\title{
Cor e qualidade formal no método de Rorschach em um grupo de crianças de 11/12 anos de idade
}

\author{
Danilo Rodrigues Silva ${ }^{1}$, Ana Sousa Ferreira \\ Universidade de Lisboa, Lisboa, Portugal
}

\section{RESUMO}

Com os resultados de alguns estudos normativos do método de Rorschach, verificou-se que os cartões ditos vermelhos, II e III, e dois dos três cartões ditos coloridos, VIII e X, suscitam a frequência mais elevada de respostas com fraca qualidade formal, levantando-se a hipótese de que a cor poderia ser responsável por tal ocorrência. Mediante o uso da versão padronizada e da versão acromática dos cartões II, III, VIII, IX e X, aplicadas a uma amostra de 80 crianças portuguesas de ambos os sexos, com idades entre 11 e 12 anos, constatou-se que apenas no cartão III se encontram indicadores irrecusáveis de que a cor vermelha está associada à frequência mais elevada de respostas de fraca qualidade formal nesse cartão. Adicionalmente, identificou-se que no cartão X a cor contribui para maior frequência de respostas de boa qualidade formal.

Palavras-chave: teste de Rorschach; crianças; cor; forma; qualidade formal.

\section{ABSTRACT - Color and form quality in the Rorschach method in a group of 11/12 year-old children}

Results from some Rorschach normative studies revealed that the said red cards II and two of the three said colored cards VIII and X elicit the highest frequency of poor form quality responses. This fact permitted the formulation of a hypothesis that color could be the cause of such an occurrence. Administration of a standardized and achromatic version of cards II, III, VIII, IX, and X to a sample of 80 Portuguese students of both sexes and aged 11 to 12 years old revealed that only with card III there is strong evidence that the color red is associated with the high frequency of poor form responses. Additionally, results showed that the color is responsible for a higher frequency of good form responses on card X.

Keywords: Rorschach test; children; color, form; form quality.

\section{RESUMEN - Color y calidad formal en el Rorschach de un grupo de niños de 11/12 años}

A partir de los resultados obtenidos con algunos estudios normativos del Rorschach, se ha verificado que las láminas rojas, II y III, y las dos láminas coloridas VIII y X se cuentan entre las cinco que proporcionan mayor frecuencia de respuestas con forma distorsionada. Este dato nos ha inducido a levantar la hipótesis de que el color podría ser la causa de la presencia acentuada de tales respuestas. La aplicación de la versión estándar y de la versión acromática de las láminas II, III, VIII, IX y X a una muestra de 80 niños portugueses, de ambos géneros y 11/12 años de edad, permitió verificar la presencia de fuertes indicadores de que el color rojo, en la lámina III, es la causa de la elevada frecuencia de formas distorsionadas allí ocurridas. Adicionalmente, cumple señalar que, en la lámina X, el color contribuye para el aumento de la frecuencia de respuestas de buena calidad formal.

Palabras clave: prueba de Rorschach; niños; color; forma; calidad formal.

O teste ou método de Rorschach é um instrumento de avaliação da personalidade constituído por dez cartões com borrões ou manchas de tinta, publicado inicialmente em 1921. Numerados no verso com algarismos romanos, cinco dos cartões são acromáticos (I, IV, V, VI e VII), dois têm a cor vermelha a par da tonalidade acromática (II e III) e três são inteiramente coloridos (VIII, IX e X). Sua aplicação individual consiste em apresentar à pessoa cada cartão, solicitando-lhe que diga o que lhe parece ser.
As respostas obtidas são em geral consideradas como de base perceptiva, muito embora sua natureza e interpretação tenham sido objeto, ao longo dos anos, de orientações teóricas diversas, desde a psicanalítica - nas suas expressões freudiana, junguiana ou hartmanniana — à cognitiva, passando pela perceptoanalítica e pela fenomenológica. Objeto de múltiplas abordagens teóricas, o Rorschach acabou por tornar-se presa de diversas escolas e sistemas e sofreu as ondas da evolução dos conceitos e das ideias. 
Na década se 1950, foi publicada a obra de Meehl (1954), que propunha a via estatística como a que melhor satisfazia as exigências de objetividade e preditividade desejáveis na psicologia clínica, uma atividade ainda jovem em que o diagnóstico psicológico tinha lugar dominante. Como este último recorria predominantemente aos instrumentos que Frank (1939) designara de "técnicas projetivas", dado o insucesso decorrente do uso dos questionários de personalidade então publicados, Meehl atestava a ineficácia do diagnóstico psicológico e, em consequência, a inadequação e inutilidade daquelas técnicas.

O alargamento do seu campo de ação para o tratamento determinou, por assim dizer, a secundarização do diagnóstico em favor da avaliação, promovida pelo behaviorismo e secundada pelo cognitivismo emergente, em detrimento do uso dos testes projetivos ${ }^{2}$. As apreciações contidas em The six mental measurements yearbook, de 1965, denunciam a fraqueza do Rorschach como teste de avaliação ou diagnóstico, mostram que o seu sucesso deriva do clínico que o utiliza, aconselhando o seu uso como entrevista, e concluem que "o Rorschach é um teste medíocre, sem valor prático em relação a qualquer dos objetivos para que é proposto pelos seus devotos" (Silva, 1983).

A perspectiva de Meehl, porém, foi também objeto de crítica por parte de autores com uma perspectiva diversa do caráter científico da psicologia clínica. Com efeito, autores com uma concepção menos cientista e mais realista da autêntica natureza da psicologia clínica, cujo objeto assume características que não são compatíveis com a objetividade nem com a preditividade, ambas próprias das ciências positivas, produziram e publicaram trabalhos que permitiram situar corretamente o diagnóstico psicológico no lugar que the compete (Silva, 1987). Refere-se aqui aos excelentes artigos de Holt (1970) e Weiner (1972; 1983), que acabaram por destacar não apenas a pertinência do diagnóstico psicológico, mas também o alargamento do seu campo e a importância nos novos domínios da psicologia da saúde e forense.

No início da década de 1970, a crise das chamadas técnicas projetivas atingira o seu máximo, interrogando-se Klopfer e Taulbee em 1976: "Será esta a última vez que aparece um capítulo sobre testes projetivos na Annual Review of Psychology? Será o Rorschach uma mancha na história da psicologia clínica?" (Silva, 1983, p. 102). Mas acrescentaram: "Se as técnicas projetivas estão mortas, algumas pessoas não receberam a mensagem. Houve mais de 500 artigos de revista referentes a técnicas projectivas, durante o período corrente de revisão (1971-1974), sem incluir vários livros publicados como novos ou edições revistas" (Silva, 1983, p. 102).

Entretanto, no fim dos anos 1960, surgiu nos Estados Unidos um homem que, em reação a esse panorama e

${ }^{2} \mathrm{O}$ leitor interessado no percurso do Rorschach, na segunda metade do século XX, lerá com proveito os capítulos de Exner \& Erdberg (2005). reconhecendo o caos em que o diagnóstico psicológico se encontrava, se propôs a fazer do Rorschach um instrumento de avaliação com bases científicas e com as características dos testes objetivos. John E. Exner Jr. começou, como escreveu no prefácio da sua obra princeps, por sugestão de Rapaport e proposta de Beck e Klopfer (Exner Jr., 1986), a analisar os cinco sistemas então vigentes no país norte-americano: Beck, Klopfer, Rapaport, Piotrowski e Hertz. Deles, extraiu os dados que lhe pareceram mais consistentes. Em resultado, veio a publicar o livro The Rorschach systems (Exner Jr., 1969). Dessa análise e do acurado trabalho de investigação que se seguiu, apareceu um novo sistema avaliativo, publicado com o título The Rorschach: a comprehensive system (Exner Jr., 1974). Como a sua designação indica, o novo Rorschach compreendia — ou, num português mais ajustado, integrava - os anteriores sistemas naqueles aspectos considerados pelo autor como sólidos e apresentava-se como um instrumento verdadeiramente estandardizado nas suas instruções de aplicação, codificação e interpretação, dotado dos requisitos de garantia e validade que nunca mais deixaram de ser aperfeiçoados ou desenvolvidos ao longo dos anos posteriores. Entre muitos outros aspectos, aprofundou-se o estudo do processo de resposta que permitiu reconhecer, entre diversos momentos fundamentais do ato de resposta, que o lugar da projeção, um conceito obsessivamente presente, era dispensável, sem grande utilidade, embora pudesse estar presente em algum momento de resposta (Exner Jr., 1986).

Esse novo instrumento soube ganhar o lugar que pretendia no campo da avaliação psicológica e ter acesso às publicações então acreditadas (Erdberg \& Exner Jr., 1984). A constituição, nos anos 1990, do Rorschach Research Council, que reuniu um conjunto de importantes colaboradores e investigadores (Exner Jr., 2003), bem como a publicação da obra Issues and methods in Rorschach research (Exner Jr., 1995), a primeira no seu gênero, foi a confirmação do alcance do objetivo formulado em 1969. Um dos grandes méritos do trabalho de Exner Jr. e de sua equipe foi ainda o impulso que deu para o desenvolvimento de estudos normativos em diversos países, o que, sob a égide de Erdberg e Schaffer, veio a dar lugar, em 2007, à publicação do Suplemento 1 do Journal of Personality Assessment (Erdberg \& Shaffer, 2007).

Os estudos e as investigações continuaram a surgir, segundo critérios bem estabelecidos e o desenvolvimento de novos meios e técnicas, dotando o Rorschach de crescente credibilidade, estendendo-o por diversas latitudes e longitudes. Um dos frutos mais ricos, surgido há poucos anos, foi o herdeiro do Comprehensive System, denominado de Rorschach Performance Assessment System (Meyer, Viglione, Mihura, Erard, \& Erdberg, 2011), um título que traduz exemplarmente a transformação produzida pela atitude científica no modo de avaliação da personalidade individual e o testemunho do imenso trabalho e da isenção científica que perpassa pela sua construção (Silva, 2015). 


\section{Cor e qualidade formal}

Enquanto instrumento de avaliação, a utilização do Rorschach implica o estabelecimento de dados normativos que consistem na frequência com que ocorrem determinadas características de respostas, entre as quais estão a localização do objeto visto (no todo ou em parte da mancha), o conteúdo (um animal, uma árvore) e a qualidade formal (FQ) dos objetos referidos nas respostas - que podem ser formalmente compatíveis (boa forma) ou incompatíveis (má forma) com a mancha ou parte dela, em que são identificados.

O estudo normativo do Rorschach, em Portugal, foi realizado com 359 crianças de ambos os sexos, distribuídas por cinco grupos com idades entre 6 e 10 anos, provenientes de diversas escolas de Lisboa. Ele foi publicado em 2007 no suplemento I do Journal of Personality Assessment (Silva \& Dias, 2007b). Com base nesse estudo e dada a diferença acentuada encontrada entre os valores de FQ obtidos, claramente inferiores aos conhecidos de Exner Jr. e Weiner (1995), procedeu-se à elaboração da respectiva tabela de FQ, que permitiu determinar a ordem segundo a qual se distribuem os dez cartões do Rorschach: II, III, VIII, VII, X, IX, IV, VI, I, V (Silva \& Dias, 2007a). Como se pode ver, quatro dos cinco cartões que contêm cor se acham entre os primeiros cinco cuja frequência de FQ é mais elevada. São os cartões II, III, VIII e X. O cartão VII é o único cartão acromático cujo número de respostas de má forma é elevado, ocupando o quarto lugar na ordem decrescente referida. Esse dado suscitou, naturalmente, a questão: será a cor, nessa amostra e idade, causa do aumento da distorção perceptiva nas normas portuguesas do Rorschach para crianças?

A pesquisa sobre o efeito da cor na produção de respostas remonta à publicação de um artigo de Exner Jr., em 1962, em que demonstrou, com uma amostra de jovens adultos universitários, que a cor determina o aumento da produção de respostas (Exner Jr., 1962).

Num novo estudo que empreendemos sob sua sugestão, destinado a verificar se o maior número de respostas frequentemente suscitado pelo cartão X se devia ao seu caráter fragmentado, como pretendiam Ainsworth e Klopfer (1954), ou ao fato de ser colorido, ficou confirmado que a cor era o elemento responsável por tal aumento. Nessa mesma investigação, que utilizou uma amostra de 40 crianças de 9 e 10 anos de ambos os sexos, verificou-se que a cor, ao contrário do esperado, não tem qualquer efeito na produção de respostas (Silva, 2002). Isso foi posteriormente confirmado com idênticas amostras de crianças de 5 e 6 anos e 11 e 12 anos, constatando ligeira redução da produção de respostas nos cartões coloridos, tanto mais notada à medida que essa produção aumentava nos restantes cartões (Silva \& Marques, 2008a; 2008b). Esse aspecto acha-se, aliás, expresso pelo decréscimo dos valores de quociente afetivo nos dados normativos para crianças e adolescentes de Exner Jr. e Weiner (1995), os quais descem de 0,88 e 0,87 , nos 5 e 6 anos, para 0,602 e 0,65 , nos 11 e 12 anos. Nos dados normativos portugueses, essa redução também foi observada, mas de modo menos acentuado; foi mais visível o aumento da produção no restante dos cartões.
Tais fatos permitem destacar a dimensão do desenvolvimento do efeito da cor na produção de respostas, um aspecto que não foi devidamente considerado nos estudos do Rorschach de crianças em idade escolar (Silva, 2009). Foi na obra de Rausch e Boizou (1977) que se encontraram os comentários mais ajustados em função do que aqui fica dito. Com efeito, as autoras afirmam, de modo muito claro, que a valoração interpretativa da cor em protocolos de crianças deve apoiar-se não na codificação das respostas nem no correspondente significado que tem nos protocolos de adultos, mas na interpretação qualitativa assente na verbalização e em outras formas de reação à presença da cor veiculadoras da reação afetiva e emocional inerente. Nessa mesma linha, uma variável como a de quociente afetivo, ou RC\%, não tem significado em protocolos de crianças. Nota-se, no entanto, que esse dado, verificado em Lisboa e compatível com os resultados de Exner Jr. (1962) já referidos, não é universal, como mostra o estudo realizado na cidade de Quelimane (Moçambique) e publicado por Silva e Ferreira (2011), cujos resultados, no grupo acima de 16 anos, não confirmam os já citados.

Os estudos normativos antes indicados, o reconhecimento de que a FQ está mais frequentemente presente em quatro dos cinco cartões do Rorschach com cor e o nosso empenho simultâneo na análise do efeito da cor na produção de respostas justificam o título deste trabalho, cujo objetivo foi apenas verificar se a cor pode estar ligada à referida distorção perceptiva elevada.

\section{Método}

\section{Participantes}

A amostra foi constituída por 80 crianças de 11 e 12 anos de idade, com igual número de ambos os gêneros. Desse total, 51 (63,7\%) tinha 11 anos e 29 (36,3\%) 12 anos de idade, todas provenientes de escolas de Lisboa (Portugal) e arredores. As idades dos participantes do estudo eram compatíveis com os respectivos anos de escolaridade, meio de salvaguardar a normalidade do rendimento escolar e do nível intelectual e comportamental das crianças. Estas foram subdivididas em dois grupos de igual número de participantes (40 em cada), conforme a versão (cromática ou acromática) do Rorschach a que foram submetidas. Todas elas foram examinadas pelo primeiro autor, dada a ausência de um colaborador disponível, como seria desejável.

\section{Materiais}

Com o intuito de examinar devidamente o efeito da cor na produção de respostas de má FQ, aplicaram-se os cartões II, III, VIII, IX e X, na sua versão original cromática e numa versão acromática. As instruções de aplicação foram as preconizadas pelo sistema compreensivo de Exner Jr. (2003). De acordo com a instrução desse estudioso, os protocolos interpretativamente válidos devem ter no mínimo 14 respostas, no entanto no presente trabalho foram considerados os protocolos cuja produção alcançou 
pelo menos sete respostas, visto que não se tratava propriamente de uma questão de interpretação do caso, mas do mínimo de respostas requerido para o exame do tema em foco, tendo em vista os cinco cartões apresentados. Foi invalidado qualquer protocolo com eventual recusa de resposta aos cartões do Rorschach.

\section{Procedimentos}

\section{Coleta de dados}

Como habitualmente, foi necessário solicitar a autorização do Ministério da Educação de Portugal para concretizar este plano de estudo. De posse da autorização, contataram-se diversas escolas para obter o consentimento e a colaboração do respectivo diretor. Precisou-se, depois, da autorização dos pais ou cuidadores das crianças, para se proceder à aplicação da prova de Rorschach, no que, gratamente, se obteve a colaboração dos diretores.

Ao longo do estabelecimento inicial da relação com a criança, realizada na própria escola, comunicando-lhe o que se iria fazer, perguntava-se-lhe se aceitava participar do estudo. O exame só teria lugar se a resposta fosse afirmativa, modo de nos assegurarmos da devida voluntariedade. Em apenas um caso a resposta foi negativa.

A aplicação da série cromática ou acromática do Rorschach foi efetuada de maneira aleatória, de forma a obter igual número de crianças, de ambos os gêneros, em cada um dos dois grupos.

Os protocolos foram integralmente codificados pelo primeiro autor. Vinte deles foram também codificados por um colega docente de outra faculdade, com o objetivo de avaliar a garantia intercotadores ou a concordância de codificação. Esta última, expressa em termos de percentagem de acordo, alcançou o valor de 91,1\%.

\section{Análise dos resultados}

As variáveis consideradas foram as relacionadas à $\mathrm{FQ}$ do sistema compreensivo do Rorschach, com os seguintes símbolos: + , símbolo de uma forma comum, mas elaborada; $o$, símbolo da resposta com forma comum; -, símbolo de uma forma má ou distorcida; $u$, símbolo de uma forma de contornos adequados à resposta dada, mas de ocorrência rara (Exner Jr., 2003). A essas variáveis, juntou-se a variável XA, introduzida por Exner Jr. e indicadora da forma adequada alargada, que resulta da soma das respostas com FQ+, FQo e FQu (Exner Jr., 2003).

A primeira análise estatística dos resultados centrouse no cálculo de correlações entre os grupos pareados, para avaliar os níveis de correlação entre a variável FQ- e a variável XA. Para efeito, levaram-se em conta os cartões do Rorschach individualmente e agrupados em dois conjuntos: os cartões II+III e os cartões VIII+IX+X. Esta última distinção obedeceu à ideia de que a cor vermelha, com a tonalidade cinzenta escura (conjunto II + III), poderia determinar efeitos diversos dos suscitados pelo conjunto VIII $+\mathrm{IX}+\mathrm{X}$, em que o elemento acromático está praticamente ausente.

Em segundo lugar, procedeu-se à análise das diferenças entre as médias dos grupos em função das variáveis FQ- e XA. Calcularam-se as médias e os desvios-padrão de cada variável, e a significância das diferenças foi avaliada com o teste de igualdade de valores médios, usando a estatística $t$ de Student $(p \leq 0,05)$. Complementarmente, determinou-se ainda a medida da dimensão do efeito $d$ de Cohen, para avaliar o grau em que o fenômeno está presente na população.

Por fim, teve lugar a comparação entre médias de respostas dadas aos cartões nas versões cromática e acromática, tendo-se em vista as variáveis FQo, FQ-, FQu e XA. Realizou-se a Análise de Variância (ANOVA) a um fator. Para finalizar, foram ainda determinados a medida de dimensão do efeito, o eta quadrado parcial $\left(\eta_{\mathrm{p}}^{2}\right)$ e a potência de teste.

\section{Resultados}

Em conformidade com o objetivo primordial do estudo, determinar em que medida o valor de FQ- pode estar relacionado com o caráter cromático dos cartões do Rorschach, começamos por apresentar as correlações entre aquela variável e a variável XA. Embora essa medida possa parecer supérflua ou gratuita, os resultados produzidos mostraram-se de interesse pelo que puderam revelar e se comentará em sequência. Estão apresentados na Tabela 1.

Em todos os cartões do Rorschach averiguados, as relações entre FQ- eXA são, como seria de esperar, inversas

Tabela 1

Variáveis F-e XA\% na amostra total (n=80) e correlações dos resultados nos cartões II, III, VIII, IX, X e II+III e VIII+IX+X do Rorschach

\begin{tabular}{|c|c|c|c|c|c|c|}
\hline \multirow{2}{*}{$\begin{array}{l}\text { Variável Rorschach } \\
\text { Pares de F- e XA }\end{array}$} & \multicolumn{2}{|c|}{ F- } & \multicolumn{2}{|c|}{ XA } & \multirow{2}{*}{ Correlação } & \multirow{2}{*}{ Sig. } \\
\hline & Média & DP & Média & DP & & \\
\hline II F- e II XA & 1,13 & 0,83 & 1,16 & 0,83 & $-0,52$ & $<0,01$ \\
\hline III F- e III XA & 0,95 & 0,86 & 1,74 & 1,27 & $-0,62$ & $<0,01$ \\
\hline VIII F- e VIII XA & 0,89 & 0,93 & 1,59 & 1,33 & $-0,58$ & $<0,01$ \\
\hline IX F-e IX XA & 1,08 & 0,98 & 1,23 & 0,99 & $-0,47$ & $<0,01$ \\
\hline X F- e X XA & 1,48 & 1,14 & 2,03 & 1,49 & $-0,16$ & 0,15 \\
\hline II+III F- e II+III XA & 2,08 & 1,24 & 2,90 & 1,63 & $-0,05$ & $<0,01$ \\
\hline VIII+IX+X F-e VIII+IX+X XA & 3,44 & 2,07 & 4,84 & 2,73 & $-0,24$ & 0,03 \\
\hline
\end{tabular}

Nota: $\mathrm{DP}=$ desvio-padrão; Sig.=nível de significância. 
e altamente significativas, com exceção do cartão X, cujo valor não atingiu o nível mínimo de significância. É de notar a indicação de que no cartão X o valor de FQ- se aproxima do de XA, mantendo relação inversa. Daí que, ao nível dos três cartões coloridos juntos, a relação aponta ser inversa e estatisticamente significativa. Observa-se desde já que a consideração da junção dos cartões II e III e dos cartões VIII, IX e X não acusou, nesse nível, a presença de resultados manifestamente diversos ao nível da análise das correlações, como se conjecturou antes.

A seguir foram sistematizados, para cada cartão do Rorschach, os dados descritivos (médias e desvios-padrão) e a comparação de médias das variáveis XA e FQ-, bem como o nível de significância associado a essas diferenças e respectivas dimensões do efeito, medidas pelo $d$ de Cohen. Esses achados encontram-se na Tabela 2.

Os resultados exibiram a existência de diferenças estatisticamente significativas entre as respostas aos cartões III, VIII e X e entre os grupos de cartões II+III e VIII+IX+X. Tais diferenças não são estatisticamente significativas nos cartões II e IX, cujos valores de FQ- são mais altos, razão pela qual as médias das diferenças são mais baixas. As médias de XA e FQ- nos cartões II e IX são respectivamente 1,16 e 1,13 e 1,23 e 1,08. Esses resultados acompanham o reconhecimento geral de que os cartões II e IX dão lugar a um número elevado de $\mathrm{FQ}$-, sendo por isso tidos como cartões mais difíceis.

Em sequência, tem-se a análise dos resultados de cada variável de FQ: FQo, FQ-, FQu e XA, cartão por cartão, levando em conta a versão cromática e acromática deles, objetivo primordial deste estudo. A Tabela 3 ilustra esse conjunto de evidências empíricas. Apresentam-se as médias de FQo em cada cartão, nas suas versões cromática e acromática, seguidas da estatística $F$, do respectivo nível de significância e da medida de dimensão do efeito $\left(\eta_{\mathrm{p}}^{2}\right)$.

Como se pode ver, apenas no cartão $\mathrm{X}$ a qualidade cromática contribuiu para um número significativamente mais elevado de respostas FQo, em comparação com o obtido na sua versão acromática. Nesse cartão, a potência de teste é de nível aceitável e reforça a influência do seu cromatismo na ocorrência mais elevada de respostas de boa forma quando comparado com a versão acromática. Trata-se de um pormenor que merece ser destacado, já que ele não acontece em nenhum outro dos cinco cartões com cor.

No cartão II, a diferença entre médias de FQo mostrou ser tendencialmente significativa, mas as respostas dadas registraram os mais baixos valores obtidos nos cinco cartões. Nos cartões III, VIII e IX, as diferenças entre médias de respostas FQo entre versões cromática e acromática são pequenas, não significativas do ponto de vista estatístico, aspecto que permite registrar que a cromaticidade não favorece, particularmente, a ocorrência de respostas de boa forma. Salienta-se, pois, que apenas nos cartões II e X as diferenças identificadas favoreceram a versão cromática, contribuindo para um maior número de respostas de boa forma, ao nível mínimo de significância estatística (atingindo $p=0,10$ no cartão II, e $p=0,05$ no cartão X). No entanto no cartão II o valor de FQo, na versão cromática, foi um dos mais baixos, enquanto no cartão X esse valor foi o mais alto.

A mesma estrutura de dados é exibida na Tabela 4, porém agora com respeito à variável FQ-.

Tabela 2

Resultados e comparação estatística das variáveis XA e F-nos cartões II, III, VIII, IX, X, II+III e VIII+IX+X do Rorschach (n=80)

\begin{tabular}{|c|c|c|c|c|c|c|}
\hline $\begin{array}{l}\text { Estatística } \\
\text { Pares de variáveis Rorschach }\end{array}$ & Média & DP & t de Student & g.l. & Sig. & $d$ de Cohen \\
\hline II XA e II F- & 0,04 & 1,45 & 0,23 & 79 & 0,82 & 0,03 \\
\hline III XA e III F- & 0,79 & 1,92 & 3,67 & 79 & $<0,01$ & 0,41 \\
\hline VIII XA e VIII F- & 0,70 & 1,83 & 3,42 & 79 & $<0,01$ & 0,38 \\
\hline IX XA e IX F- & 0,15 & 1,69 & 0,79 & 79 & 0,43 & 0,09 \\
\hline X XA e X F- & 0,55 & 2,02 & 2,44 & 79 & 0,02 & 0,27 \\
\hline II+III XA e II+III F- & 0,83 & 2,54 & 2,91 & 79 & $<0,01$ & 0,33 \\
\hline VIII+IX+X XA e VIII+IX+X F- & 1,40 & 3,80 & 3,30 & 79 & $<0,01$ & 0,37 \\
\hline
\end{tabular}

Nota: $\mathrm{DP}=$ desvio-padrão; g.l.=graus de liberdade; Sig.=nível de significância.

Tabela 3

Análise de variância a um fator das respostas FQo dadas às versões cromática e acromática de cada cartão do Rorschach ( $n=80$ )

\begin{tabular}{lcccccccc}
\hline Grupo & \multicolumn{2}{c}{ Cromático $(n=40)$} & \multicolumn{2}{c}{ Acromático $(n=40)$} & \multicolumn{3}{c}{ Estatística } \\
\cline { 2 - 8 } Cartões & Média & DP & Média & DP & $F$ & Sig. & $\eta^{2}$ Potência de teste \\
\hline II & 0,65 & 0,70 & 0,40 & 0,55 & 3,28 & 0,07 & 0,04 & 0,43 \\
III & 1,10 & 1,03 & 1,33 & 0,92 & 1,07 & 0,31 & 0,01 & 0,18 \\
VIII & 0,75 & 0,44 & 0,73 & 0,68 & 0,04 & 0,85 & $<0,01$ & 0,05 \\
IX & 0,32 & 0,66 & 0,32 & 0,62 & 0,00 & 1,00 & $<0,01$ & 0,05 \\
X & 1,22 & 1,00 & 0,75 & 0,93 & 4,79 & 0,03 & 0,06 & 0,58 \\
\hline
\end{tabular}

Nota: DP=desvio-padrão; F=estatística F; Sig.=nível de significância; $\eta_{\mathrm{p}}^{2}=$ medida de dimensão do efeito. 
A análise desses resultados revelou ainda apenas uma diferença estatisticamente significativa entre as médias de respostas dadas ao cartão III, com elevada potência de teste, a favor da versão cromática. Isso significa que a presença da cor vermelha suscita número significativamente elevado de respostas FQ-, em comparação com o número suscitado pela sua versão acromática. Trata-se de um dado bastante interessante, sobretudo diante dos achados referentes ao cartão II, no qual o vermelho também está presente e as médias de FQ-, em ambas as versões, cromática e acromática, estão próximas uma da outra. $\mathrm{O}$ achado permite considerar que no cartão II a frequência elevada de respostas FQ- não se deve apenas nem sobretudo à presença da cor vermelha. Enquanto isso, no cartão III a cor se associa fortemente à elevação de FQ- e a sua ausência favorece a FQo. No cartão II, a ausência da cor não altera a frequência da qualidade formal negativa das respostas, que se mantém idêntica e elevada em ambas as versões. Por conseguinte, a conhecida frequência elevada de FQ-, no cartão II, terá origem em outro elemento que não apenas a cor vermelha. É de destacar o valor relativamente elevado da potência de teste da diferença encontrada no cartão III.

Um pormenor interessante, que suscita mais consistência no resultado analisado, decorre da comparação desses resultados com os da variável FQo. Ao passo que no cartão III a versão acromática favoreceu o aumento de FQo $(1,33)$ e reduziu a frequência de FQ- $(0,70)$, a versão cromática não impediu que a média de FQo alcançasse um nível próximo da de FQ- $(1,10 / 1,20)$. Assim, enquanto a frequência elevada de FQ-, na versão cromática do cartão III, pareceu dever-se prioritariamente à presença do vermelho, a frequência idêntica dessa variável no cartão II teve origem mais complexa. Um aspecto que pode contribuir para avaliar o alcance dessa última afirmação reside no fato de que, ao mesmo tempo em que no cartão III a frequência de FQ- se aproximou da de FQo, no cartão II a frequência de FQo foi bastante baixa e se distanciou bastante da de FQ-.

Em complemento a esses achados, têm-se os dados referentes à variável $\mathrm{FQu}$, os quais compõem a Tabela 5 . O exame dessa tabela possibilita verificar, com facilidade, que nos cartões II e III a média de respostas FQu dadas à versão acromática foi mais alta do que a de respostas dadas à versão cromática e que a média do cartão II foi mesmo significativamente mais alta.

Nesses dois cartões vermelhos ocorreram as médias mais baixas de FQu na versão cromática, indicando, de forma clara, que se trata de cartões em que predominam o FQ- e/ou o FQo. Inversamente, nos cartões coloridos VIII, IX e X, as médias de $\mathrm{FQu}$, na versão cromática, tenderam a ser mais altas do que as da versão acromática. Um dado bem visível foi que os cartões II e III não são favoráveis à ocorrência do tipo $u$ de FQ, particularmente na sua versão cromática. Por outro lado, o fato de no cartão II haver diferença estatisticamente significativa entre médias de respostas $\mathrm{FQu}$ dadas nas duas versões, favorável à versão acromática, sugere que o determinante da diferença no cartão II não era idêntico ao do cartão III, como parece ser também o caso da ocorrência elevada de FQ- nesses mesmos cartões. Um aspecto que deve ser salientado com respeito à ocorrência de respostas de boa FQ nesse cartão é que, entre os cinco cartões com cor considerados neste estudo, o cartão II foi aquele no qual se deu o menor número de respostas de boa FQo, quer na versão cromática, quer na acromática. Apenas no cartão IX o valor de FQo foi menor do que o do cartão II em ambas as versões.

Tabela 4

Análise de variância a um fator das respostas FQ- dadas às versões cromática e acromática de cada cartão do Rorschach ( $n=80$ )

\begin{tabular}{lcccccccc}
\hline Grupo & \multicolumn{2}{c}{ Cromático $(n=40)$} & \multicolumn{2}{c}{ Acromático $(n=40)$} & \multicolumn{3}{c}{ Estatística } \\
\cline { 2 - 9 } Cartóes & Média & DP & Média & DP & F & Sig. & $\eta_{\text {p }}^{2}$ & Potência de teste \\
\hline II & 1,20 & 0,94 & 1,05 & 0,71 & 0,69 & 0,41 & 0,01 & 0,13 \\
III & 1,20 & 0,91 & 0,70 & 0,72 & 7,60 & 0,01 & 0,09 & 0,78 \\
VIII & 0,83 & 0,87 & 0,95 & 0,99 & 0,35 & 0,56 & 0,01 & 0,09 \\
IX & 1,03 & 1,14 & 1,13 & 0,79 & 0,21 & 0,65 & $<0,01$ & 0,07 \\
X & 1,58 & 1,22 & 1,38 & 1,06 & 0,65 & 0,42 & 0,01 & 0,13 \\
\hline
\end{tabular}

Nota: DP=desvio-padrão; F=estatística F; Sig.=nível de significância; $\eta^{2}=$ =medida de dimensão do efeito.

Tabela 5

Análise de variância a um fator das respostas FQu dadas às versões cromática e acromática de cada cartão do Rorschach ( $n=80$ )

\begin{tabular}{lcccccccc}
\hline Grupo & \multicolumn{2}{c}{ Cromático $(n=40)$} & \multicolumn{2}{c}{ Acromático $(n=40)$} & \multicolumn{3}{c}{ Estatística } \\
\cline { 2 - 9 } Cartóes & Média & DP & Média & DP & $F$ & Sig. & $\eta^{2}{ }_{p}$ & Potência de teste \\
\hline II & 0,45 & 0,60 & 0,83 & 0,87 & 5,02 & 0,03 & 0,06 & 0,60 \\
III & 0,43 & 0,59 & 0,63 & 0,74 & 1,77 & 0,19 & 0,02 & 0,26 \\
VIII & 1,00 & 1,06 & 0,70 & 0,82 & 2,02 & 0,16 & 0,03 & 0,29 \\
IX & 1,00 & 0,88 & 0,80 & 0,85 & 1,07 & 0,31 & 0,01 & 0,18 \\
X & 1,13 & 1,11 & 0,95 & 0,99 & 0,54 & 0,46 & 0,01 & 0,11 \\
\hline
\end{tabular}

Nota: DP=desvio-padrão; F=estatística F; Sig.=nível de significância; $\eta^{2}{ }_{\mathrm{p}}=$ medida de dimensão do efeito. 
Por fim, estão os resultados concernentes ao conjunto das variáveis de boa forma (XA), na versão cromática e acromática de cada cartão. A Tabela 6 traz esses dados.

É possível observar, de novo, o fenômeno anteriormente assinalado, referente à variável FQu. Nos cartões II e III, as médias de XA, na versão acromática, foram mais altas do que as da versão cromática. Nos cartões VIII, IX e $\mathrm{X}$, as médias das respostas $\mathrm{XA}$, na versão cromática, foram mais altas do que na acromática. Esse fato, expresso pelos respectivos valores médios, pode, no entanto, levar ao erro de considerar que nos cartões II e III a versão acromática favorece as respostas de boa $\mathrm{FQ}$, o que não é correto. Com efeito, no cartão II, os valores de boa FQ, como já se enfatizou, foram baixos nas duas versões, como foram altos os valores das respostas de má FQ. A diferença entre o cartão II e o cartão III reside em que, no primeiro, a boa FQ foi pouco frequente nas duas versões; no segundo, ela atingiu frequência mais elevada em ambas as versões.

Parece também evidente que as cores pastel dos cartões ditos coloridos favoreceram a ocorrência de boas formas, alcançando diferença estatisticamente significativa no cartão X, com potência de teste aceitável. Uma análise dos dados, contudo, permite detectar elementos que contrariam essa ideia geral.

No cartão IX, achou-se a média mais baixa de FQo $(0,32)$, sendo a do cartão VIII, de apenas 0,75 . Curiosamente, nesses dois cartões, as médias de FQu foram iguais $(1,00)$. Outro dado curioso é que, enquanto nos cartões II e III os valores de FQo foram claramente mais elevados do que os de FQu, nos cartões VIII e IX os valores de FQu foram nitidamente mais altos do que os de FQo e apenas um pouco mais baixos no cartão X. Globalmente, verifica-se, de maneira efetiva, que as médias de resposta de boa FQ foram mais elevadas na versão cromática do que na acromática, mas essa diferença não é estatisticamente significativa. Por outro lado, não se pode perder de vista que se está perante quatro dos cinco cartões com percentagens de FQ- mais elevadas e que o cartão IX figura como um dos que suscitam idêntica percentagem de distorção perceptiva, em outros estudos normativos, como se verá mais adiante. De resto, como foi possível constatar pela ordem dos cartões, em função da elevação de FQ-, nos dados normativos de crianças portuguesas (Silva \& Dias, 2007a), o cartão IX figurou em sexto lugar, logo depois do cartão X.

O último dado suscitado pela análise desses resultados e que ilustra, de modo flagrante, a diversidade de comportamento reativo nos cartões II e III e nos VIII, IX e X, já mais do que uma vez assinalado, se relaciona com a oposição da ocorrência das médias de FQ- e de XA nos dois conjuntos de cartões. Nos cartões II e III, os valores de FQ- foram mais altos na versão cromática do que na acromática, ao passo que os valores de XA foram mais elevados na versão acromática do que na cromática. Ao contrário, nos cartões VIII, IX X X, encontramos valores mais elevados de FQ- na versão acromática do que na cromática, com exceção do cartão X, e valores de XA mais elevados na versão cromática do que na acromática. Ressalta-se que, com exceção do cartão X, em que se observou diferença estatisticamente significativa em XA, favorável à versão cromática, todas as demais diferenças achadas não foram estatisticamente significativas; indicavam apenas tendência dominante. Assinala-se, de passagem, a identificação da diferença entre as reações expressas pelas diferenças entre frequências de FQ- e XA nos cartões vermelhos e nos cartões coloridos.

Nesse passo, podem ser comparadas as médias de FQo e FQ- nos vários cartões e em ambas as versões, conforme mostra a Tabela 7.

Tabela 6

Análise de variância a um fator das respostas XA dadas às versões cromática e acromática de cada cartão do Rorschach ( $n=80$ )

\begin{tabular}{lcccccccc}
\hline Grupo & \multicolumn{2}{c}{ Cromático $(n=40)$} & \multicolumn{2}{c}{ Acromático $(n=40)$} & \multicolumn{3}{c}{ Estatística } \\
\cline { 2 - 9 } Cartões & Média & DP & Média & DP & $F$ & Sig. & $\eta^{2}$ Potência de teste \\
\hline II & 1,10 & 0,87 & 1,23 & 0,80 & 0,48 & 0,49 & 0,01 & 0,11 \\
III & 1,53 & 1,34 & 1,95 & 1,18 & 2,32 & 0,13 & 0,03 & 0,32 \\
VIII & 1,75 & 1,08 & 1,42 & 1,17 & 1,66 & 0,20 & 0,02 & 0,25 \\
IX & 1,33 & 1,05 & 1,13 & 0,94 & 0,85 & 0,36 & 0,01 & 0,15 \\
X & 2,35 & 1,53 & 1,70 & 1,40 & 3,89 & 0,05 & 0,05 & 0,50 \\
\hline
\end{tabular}

Nota: $\mathrm{DP}=$ desvio-padrão; $F=$ estatística F; Sig.=nível de significância; $\eta_{\mathrm{p}}^{2}=$ medida de dimensão do efeito.

Tabela 7

Resultados descritivos de FQo e FQ- nas versões cromática e acromática dos cartões do Rorschach com cor $(n=80)$

\begin{tabular}{|c|c|c|c|c|c|c|c|c|}
\hline \multirow{3}{*}{$\begin{array}{l}\text { Grupo } \\
\text { Cartões }\end{array}$} & \multicolumn{4}{|c|}{ Cromática } & \multicolumn{4}{|c|}{ Acromática } \\
\hline & \multicolumn{2}{|c|}{ FQo } & \multicolumn{2}{|c|}{ FQ- } & \multicolumn{2}{|c|}{ FQo } & \multicolumn{2}{|c|}{ FQ- } \\
\hline & Média & DP & Média & $D P$ & Média & $D P$ & Média & $D P$ \\
\hline II & 0,65 & 0,70 & 1,20 & 0,94 & 0,40 & 0,55 & 1,05 & 0,71 \\
\hline III & 1,10 & 1,03 & 1,20 & 0,91 & 1,33 & 0,92 & 0,70 & 0,72 \\
\hline VIII & 0,75 & 0,44 & 0,83 & 0,87 & 0,73 & 0,68 & 0,95 & 0,99 \\
\hline IX & 0,32 & 0,66 & 1,03 & 1,14 & 0,32 & 0,62 & 1,13 & 0,79 \\
\hline $\mathrm{X}$ & 1,22 & 1,00 & 1,58 & 1,22 & 0,75 & 0,93 & 1,38 & 1,06 \\
\hline
\end{tabular}

Nota: DP=desvio-padrão. 
Facilmente se reconhecerá que as médias de FQ- foram mais altas em todos os casos exceto um, o do cartão III, na versão acromática, em que a média de FQo $(1,33)$ foi mais alta do que a de FQ- $(0,70)$. Esse dado permite destacar a importância da cor vermelha na frequência da distorção perceptiva das respostas a esse cartão.

\section{Discussão}

Afirmou-se, no início deste trabalho, que quatro dos cinco cartões do Rorschach, nos quais se encontra cor, figuravam entre os cinco estímulos cuja tabela de FQ continha maior número de entradas com FQ má (FQ-), segundo a seguinte ordem decrescente: II, III, VIII, VII e X. Outros dados ligados a essa mesma questão e que igualmente apontaram a ocorrência de elevado número de FQ- provêm de outros estudos normativos: o de Pires (2007) e o de Exner Jr. e Erdberg (2005). Na amostra portuguesa de adultos não pacientes, os cinco cartões com percentagens mais altas de FQ- foram os seguintes: II, III, X, IX e VII (Pires, comunicação pessoal de 15/05/2011), em grande medida coincidentes com os encontrados com crianças (Silva \& Dias, 2007b).

No grupo de 450 não pacientes, que constitui a nova amostra de Exner Jr., os primeiros cinco cartões com percentagens mais elevadas de FQ- foram: III, IX, X, IV, VIII, dos quais quatro cartões tinham cor. Em sua amostra total de 1.050 não pacientes, os cartões com ordem decrescente de FQ- consistiram nos seguintes: III, IX, VIII, VII e X (Exner Jr. \& Erdberg, 2005). Nessas quatro amostras, os cartões III e X foram constantes, os cartões VII, VIII e IX ocorreram em três das amostras, e o cartão II nas duas amostras portuguesas, mas em todas elas figuraram quatro cartões com cor, aspecto que reforça a ideia inicial de que a cor pode constituir um elemento favorecedor de distorção perceptiva. Do mesmo modo, não deixa de ser notável verificar que, embora uma daquelas amostras seja constituída por crianças dos 6 aos 10 anos e as restantes por adultos de países distantes, tenha havido tamanha sobreposição de dados.

Apenas no caso da nova amostra de 450 adultos não pacientes de Exner Jr. e Erdberg (2005), ocorreu o cartão IV, acromático, como gerador de elevado número de respostas FQ-. Vale a pena destacar a presença do cartão VII como estimulador de grande número de respostas FQ-, em três das amostras referidas. Por sua vez, a ocorrência do cartão II como indicador da mais elevada frequência de respostas FQ-, apenas nas duas amostras normativas portuguesas de crianças e adultos, é um elemento singular e digno de exame.

A análise dos resultados encontrados, tendo em consideração apenas o efeito da cor na FQ das respostas, permite-nos afirmar:

- no que se refere à variável $\mathrm{FQo}$, as suas frequências nas versões cromática e acromática de cada cartão estavam próximas, com exceção do cartão X, em que se constatou diferença estatisticamente significativa favorável à respectiva versão cromática. Nota-se que no cartão II a diferença é apenas tendencialmente significativa $(\mathrm{p}<0,10)$, muito embora o valor médio de FQo nesse cartão tenha sido baixo. O que houve de comum entre os cartões II e X foi o fato de ambos suscitarem elevado número de FQ-;

- no caso da variável FQ-, também foi encontrada apenas uma diferença estatisticamente significativa, a que enfatiza o maior número de respostas com FQ-, na versão cromática do cartão III. Seja como for, em conformidade com os dados disponíveis, é possível afirmar que a cor não deve ser tida como a causa única do aumento das respostas FQ- no Rorschach. Com efeito, tal aumento pôde ser observado, quer nas versões cromáticas, quer nas acromáticas dos cartões, o que implica que outra causa ou causas estão a atuar com esse efeito, eventualmente o caráter interciso ou fragmentado dos cartões. De igual modo, também se afiança que a cor não contribui significativamente para a ocorrência de respostas de boa $\mathrm{FQ}$, a não ser no cartão $\mathrm{X}$, mediante as variáveis $\mathrm{FQo} \mathrm{e} \mathrm{XA;}$

- a respeito das respostas $F Q u$, uma diferença estatisticamente significativa aconteceu no cartão II favorável à versão acromática. Um aspecto já apontado antes é que a presença da cor vermelha pareceu reduzir a frequência das respostas $\mathrm{FQu}$, assim como de respostas FQo, ao passo que as cores pastel as aumentaram. Com efeito, o único caso em que, tomando separadamente os cartões vermelhos e os coloridos, a soma das médias de cada variável em estudo foi maior na respectiva versão acromática foi o da variável XA, nos cartões II e III. Significa isso que, nesses cartões, o caráter acromático favoreceu a ocorrência de respostas com boa $F Q$, no que a modalidade $F Q u$ tem papel decisivo, a par da média de FQo no cartão III;

- a mesma situação da $\mathrm{FQu}$ passou-se com a variável $\mathrm{XA}$. De fato, as suas médias foram mais elevadas na versão acromática do que na cromática. O inverso aconteceu nos cartões coloridos, em que as médias de XA foram mais altas na versão cromática do que na acromática, sendo significativamente mais elevada no cartão X. Poder-se-ia sublinhar que a ausência de diferenças estatisticamente significativas entre médias de XA na versão cromática e na acromática reforça a ideia, já antes expressa, de que a cor não é um fator importante nem muito menos exclusivo com efeito na produção de respostas com distorção perceptiva. Se o cartão X suscitou uma média de XA significativamente mais alta na sua versão cromática do que na acromática, não deixou de ser certo que a média de respostas FQ-, na versão cromática, atingiu o valor de 1,58 , a mais elevada dos cinco cartões com cor, superior às médias de FQo e de FQu, 1,22 e 1,13, respectivamente. Nota-se que o cartão $\mathrm{X}$, mesmo na sua versão acromática, obteve média relativamente elevada de FQ- $(1,38)$. 
Esses pormenores levam a considerar que a distribuição equilibrada ou não da boa ou má FQ nos diversos cartões do Rorschach pode ter causas diversas que não apenas a cor ou o caráter mais ou menos fragmentado dos estímulos presentes nos cartões.

Consideremos o cartão VII, que, como anteriormente referido, ocorre em três amostras de dados normativos como um dos que suscitam maior número de respostas FQ-. Trata-se de um cartão que em geral não é contado nem no grupo dos cartões fechados nem no grupo dos cartões fragmentados. Como é de se esperar e assim se verificou, quanto mais unido ou compacto fosse o borrão de tinta, maior seria o número de respostas globais ou respostas que abrangeriam a totalidade do borrão. Ao contrário, quanto mais dividido ou fragmentado fosse esse borrão, menor seria a ocorrência de respostas globais e maior o número de respostas que abrangeriam partes do borrão. Ora, se tomarmos a ordenação dos 10 cartões do Rorschach segundo o número de respostas globais (W) ou de objetos que se localizam na totalidade do borrão de tinta, observamos que o cartão VII se situou aproximadamente no meio dessa distribuição. Com efeito, tomando como referência as amostras de 450 e 1.050 não pacientes de Exner Jr. e Erdberg (2005), o cartão VII situou-se em sexta posição, na primeira amostra, e em quinta posição na segunda.

Cabe, então, perguntar: o que é que pode determinar a elevada frequência de FQ-nesse cartão? Dado que naquelas amostras os cartões que suscitaram maior número de FQforam os III, VIII, IX e X, todos com cor e fragmentados, não sendo o cartão VII um cartão nem fragmentado nem unido, um potencial causador de tal ocorrência seria, em nosso entender, a presença da ampla área branca que a mancha abarcava, que alguns autores chegaram a interpretar como o símbolo de vazio. Nessa mesma linha, pode-se perguntar se um fenômeno semelhante não estaria na origem do elevado número de FQ- no cartão II ou mesmo no cartão IX, em que, para além da cor vermelha ou do cromatismo de todo o cartão, a presença dos espaços brancos centrais (DS5), no primeiro, e de espaços em branco na zona DS8, no segundo, não interpretados como vazio, não estaria na origem da elevada frequência de respostas FQ-. Essa é uma hipótese a se investigar.

Como conclusão, pode-se dizer que a cor é susceptível de ser apontada como determinante principal do aumento de específicos tipos de FQ na produção de respostas ao Rorschach, nos seguintes casos:

- como FQo, no cartão X;

- como FQ-, no cartão III;

- como XA, no cartão X.

Apenas no cartão III a cor vermelha pareceu determinar o aumento das respostas de má FQ. Tal fato não se verificou no cartão II, no qual a cor vermelha também estava presente, mas não foi acompanhada duma redução daquela $\mathrm{FQ}$, na versão acromática. $\mathrm{Na}$ versão acromática do cartão III, a FQo atingiu, por sinal, uma das duas médias mais elevadas de todos os cartões estudados. Os resultados obtidos e a sua análise permitiram invalidar a hipótese de que a cor, excetuado o cartão III, é o determinante principal ou mesmo o mais importante para a alta frequência de respostas de má FQ observada nos cartões com cor. É também possível que elementos de natureza meramente cultural possam estar na origem de tais frequências, como parece ser o caso do cartão II, que, nas amostras portuguesas de crianças e adultos, apareceu como aquele com maior frequência de respostas FQ-e que, nas amostras norte-americanas referidas, se situou no oitavo lugar na ordem decrescente daquele modo de FQ.

Ao terminar a escrita deste trabalho, invocam-se os resultados recentemente alcançados e publicados referentes ao estudo do efeito da cor na produção de respostas ao Rorschach (Silva \& Ferrreira, 2014). Este último estudo culmina numa longa investigação sobre aquela temática, a qual confirmou, por um lado, que a cor é o principal elemento associado ao aumento do número de respostas aos cartões coloridos, VIII, IX e X, e revelou, por outro, que tal efeito não tinha lugar nos protocolos de crianças. A pesquisa citada teve por objetivo estabelecer em que período etário se operava a ocorrência do aumento da produção de respostas àqueles cartões, tendo-se estabelecido a idade de 15 e 16 anos.

Perante esse dado de natureza desenvolvimental, considera-se adequado repetir esta mesma investigação, relativa à possível relação entre cor e $\mathrm{FQ}$, com um grupo de adolescentes ou jovens adultos, pois no grupo estudado a presença da cor ainda não determinou o aumento da produção de respostas.

\section{Referências}

Ainsworth, M. D., \& Klopfer, B. (1954). Quantitative analysis. Em B. Klopfer, M. D. Ainsworth, W. G. Klopfer \& R. R. Holt (1954). Developments in the Rorschach technique: technique and theory. v. 1. (pp. 249-316). Nova York: Harcourt, Brace \& World.

Cohen, J. (1988). Statistical Power Analysis for the behavioral Sciences. Hillsdale, N. J., Lawrence Erlbaum Associates.

Erdberg, P. \& Exner Jr., J. E. (1984). Rorschach assessment. Em G. Goldstein \& M. Hersen (Eds.). Handbook of psychological assessment. Nova York: Pergamon Press.

Exner Jr., J. E. (1962). The effect of colour on productivity in cards VIII, IX, X of the Rorschach. Journal of Projective Techniques, $26(1), 30-33$. doi: $10.1080 / 08853126.1962 .10381074$ 
Exner Jr., J. E. (1969). The Rorschach systems. Nova York: Grune \& Stratton.

Exner Jr., J. E. (1974). The Rorschach: a comprehensive system. v. 1. Nova York: John Wiley \& Sons.

Exner Jr., J. E. (1986). The Rorschach: a comprehensive system. v. 1. 2. ${ }^{a}$ edição. Nova York: John Wiley \& Sons.

Exner Jr., J. E. (1995). Issues and Methods in Rorschach Reserarch. New Jersey: Lawrence Erlbaum Associates.

Exner Jr., J. E. (2003). The Rorschach: a comprehensive system — basic foundations and principles of interpretation. v. 1. $4^{\mathrm{a}}$ edição. Nova Jersey: John Wiley \& Sons.

Exner Jr., J. E. \& Erdberg, P. (2005). The Rorschach: a comprehensive system. v. 2. $3^{\text {a }}$ edição. Nova Jersey: John Wiley \& Sons.

Exner Jr., J. E., Weiner, I. B. (1995). The Rorschach: a comprehensive system — assessment of children and adolescents. v. 3. $2^{\mathrm{a}}$ edição. Nova York: John Wiley \& Sons.

Erdberg, P., Shaffer, T. W. (2007). Special Issue: International Reference Samples for the Rorschach Comprehensive System. Journal of Personality Assessment, 89, Supplement 1.

Frank, L. K. (1939). Projective methods for the study of personality. Journal of Psychology, 8(2), 389-413. doi: 10.1080/00223980.1939.9917671

Holt, R. R. (1970). Yet another look at clinical and statistical prediction or is clinical psychology worthwhile? American Psychologist, 25(4), 337-349. doi: 10.1037/h0029481

Meehl, P. (1954). Clinical versus statistical prediction: a theoretical analysis and a review of the evidence. Minneapolis: University of Minnesota Press.

Meyer, G. J., Viglione, D. J., Mihura, J. L, Erard, R. E., Erdberg, P. (2011). Rorschach Performance Assessment System Administration, Coding, Interpretation, and Technical Manual. Rorschach Performance Assessment System, LLC.

Pires, A. A. (2007). Rorschach comprehensive system data for a sample of 309 adult nonpatients from Portugal. Journal of Personality Assessment, 89(Suppl. 1), S124-S130. doi: 10.1080/00223890701583408

Rausch, T. N., Boizou, M. F. (1977). Le Rorschach en clinique infantile l'imaginaire et le réel chez l'enfant. Paris: Dunod.

Silva, D. R., Fernandes, I. B. (1985). Notícia sobre o $11^{\circ}$ Congresso Internacional do Rorschach e Métodos Projectivos. Barcelona, 1984. Revista Portuguesa de Psicologia, 22, 173-178.

Silva, D. R. (1987). Sobre diagnóstico psicológico. Jornal de Psicologia, 6(5), 14-16.

Silva, D. R. (2002). The effect of color on productivity on card X of the Rorschach. Rorschachiana, 25, 123-138. doi: 10.1027/1192-5604.25.1.123

Silva, D. R. (2009). A função da cor na produção de respostas ao Rorschach até ao final da adolescência. Revista Iberoamericana de Diagnóstico e Avaliação Psicológica, 28(2), 149-165.

Silva, D. R. (2011a). Color y producción de respuestas al Rorschach: ensayo de interpretación. Revista de la Sociedad Española del Rorschach Y Metodos Proyectivos, 24, 26-33.

Silva, D. R. (2011b). Role of color on the production of responses in children's Rorschach protocols. Rorschachiana, 32(2), 183-198. doi: $10.1027 / 1192-5604 / \mathrm{a} 000021$

Silva, D. R. (2015). O Sistema de Avaliação do Desempenho no Rorschach (SADR): aplicação, codificação e interpretação, manual técnico (Rorschach performance assessment system administration, coding, interpretation, and technical manual). Revista Iberoamericana de Diagnóstico e Avaliação Psicológica, 40(2), 124-136.

Silva, D. R., \& Dias, A. M. (2007a). A propósito de la elaboración de una Tabla de Calidad Formal del Rorschach de una muestra de niños portugueses. Revista de la Sociedad Española del Rorschach y Metodos Proyectivos, 20, 34-38.

Silva, D. R., \& Dias, A. M. (2007b). Rorschach comprehensive system data for a sample of 359 Portuguese children at five ages. Journal of Personality Assessment, 89(S1), 131-141.

Silva, D. R., \& Ferreira, A. S. (2011). Cor e produção de respostas ao Rorschach: um estudo transcultural. Paideia, 21(49), 149-156. doi: http://dx.doi.org/10.1590/S0103-863X2011000200002

Silva, D. R., \& Ferreira, A. S. (2014). The effect of color on the production of responses to Rorschach cards VIII, IX and X in age groups of 11-12 and 15-16 years. Journal of Personality Assessment, 96(4), 426-431. doi: 10.1080/00223891.2013.876426

Silva, D. R., \& Marques, L. G. (2008a). A cor e o efeito inibitório de respostas no Rorschach de crianças. Psychologica, 48, 5-24.

Silva, D. R., \& Marques, L. G. (2008b). L'effet de la couleur sur la production de réponses au Rorschach chez des enfants non consultants. Une étude génétique. Psychologie Clinique et Projective, 14, 221-232. doi: 10.3917/pcp.014.0221

Weiner, I. B. (1972). Does psychodiagnosis have a future? Journal of Personality Assessment, 36(6), 534-546. doi: 10.1080/00223891.1972.10119809

Weiner, I. B. (1983). The future of psychodiagnosis revisited.Journal of Personality Assessment, 47(5), 451-461. doi: 10.1207/s15327752jpa4705_1

\section{Sobre os autores}

Danilo Rodrigues Silva é Doutor em Psicologia (Avaliação Psicológica) pela Universidade de Lisboa, professor catedrático aposentado da mesma universidade, investigador do Centro de Investigação em Psicologia da Universidade de Lisboa, presidente da Associação Iberoamericana de Diagnóstico e Avaliação Psicológica e membro correspondente da Academia das Ciências de Lisboa.

Ana Sousa Ferreira é Doutora em Matemática pela Universidade Nova de Lisboa, professora associada na Faculdade de Psicologia da Universidade de Lisboa e membro da Business Research Unit dessa faculdade. 